\title{
Microsurgical vasovasostomy for the treatment of intractable chronic scrotal pain after vasectomy
}

\author{
Xiang-An Tu ${ }^{1, *}$, Liang Zhao ${ }^{1, *}$, Liang-Yun Zhao ${ }^{1}$, Cai-Mei Zhou ${ }^{1}$, Jin-Tao Zhuang ${ }^{2}$, Ji-Quan Zhao ${ }^{2}$, \\ Kun-Long $\mathrm{Lv}^{2}$, Xiang-Zhou Sun ${ }^{2}$, Shao-Peng Qiu ${ }^{2}$ and Chun-Hua Deng ${ }^{2}$
}

Asian Journal of Andrology (2013) 15, 850-851; doi:10.1038/aja.2013.76; published online 8 July 2013

Dear Editor,

We present herein two rare cases of intractable chronic scrotal pain after vasectomy. The patients were effectively treated with microsurgical vasovasostomy (MVV). We also discuss the possible aetiologies of the pain and other surgical options.

Vasectomy was once the most common method of permanent contraception for men in both China and worldwide. One particularly distressing complication after vasectomy is chronic scrotal pain, which is defined as intermittent or constant, unilateral or bilateral scrotal pain for $\geqslant 3$ months. The pain is intense enough to interfere with the patient's daily activities and prompts him to seek medical attention. ${ }^{1}$ Although its aetiology remains unclear, epididymal congestion, painful sperm granulomas, vascular stasis and nerve impingement have been postulated as possible aetiologic factors. ${ }^{2}$ Non-surgical options have been used successfully to treat chronic scrotal pain after vasectomy, including scrotal support, thermal therapy, limiting activity, non-steroidal anti-inflammatory drugs, narcotic analgesics, antibiotics, neuroleptics, spermatic cord nerve block, biofeedback and psychiatric evaluation. Surgical options include reversal of the vasectomy, microsurgical spermatic cord denervation, granuloma excision, epididymectomy and orchidectomy. The microsurgical techniques used for vasectomy reversal have changed significantly in the past decade, culminating in the standard surgical procedures used today, and its indications include a desire to have more children (remarriage or after the death of a child), treatment of post-vasectomy pain and treatment of obstructive azoospermia due to traumatic or iatrogenic injury of vas deferens. ${ }^{3}$ To our knowledge, we report the first cases of the use of MVV for the treatment of intractable chronic scrotal pain after vasectomy in a Chinese hospital.

The 72-year-old and 49-year-old men presented with a more than 20 -year history of intractable, chronic scrotal pain after vasectomy. They had consulted various urologists and had undergone numerous attempted therapies in other hospitals. They reported a history of vasectomy more than 30 years and 20 years previously, respectively. They did not have any histories of haematuria, haematospermia, lower urinary tract symptoms, epididymitis, prostatitis or testicular trauma. Their physical examination was unremarkable, and both the secondary sexual characteristics and genital examination were normal. The testes were descended bilaterally and normal in size and consistency. The caput epididymides exhibited dilatation and tenderness. The vasa deferentia were palpated for painful lumps at the vasectomy sites. Digital rectal examination was unremarkable for prostatic abnormalities. Each patient underwent Doppler ultrasonography of the testes and urinary tract, urinalysis, urine culture and spermiogram to exclude primary or secondary causes of pain, including intratesticular infection, tumours and ureteral lithiasis.

At our initial consultation, the patients were asked to complete a pain and psychological questionnaire, which included pain, depression and anxiety scores. The pain score (Visual Analogue Scale) was in the form of an 11-point numerical rating score with 0 representing 'no pain' and 10 representing the 'worst possible pain'. The patients' preoperative pain scores were 5 and 6 points, respectively. The depression scores (Self-rating Depression Scale) were in the form of an 80-point numerical rating score; a score less than 50 indicated 'normal', and a score greater than 50 indicated 'depression'. The depression scores of the two patients were 35 and 38 points, respectively. The anxiety scores (Self-rating Anxiety Scale) were in the form of an 80-point numerical rating score; scores less than 50 were considered to indicate 'normal', whereas scores greater than 50 indicated 'anxiety'. The anxiety scores of the two patients were 33 and 32 points, respectively. Spermatic cord block was performed once for each patient with $6 \mathrm{ml}$ of $1 \%$ lidocaine and $1 \mathrm{ml}$ of methylprednisolone (40 mg). The patients had 3 and 7 days of complete pain relief after the blockade, respectively. The study protocol was approved by the Ethical Committee of the First Affiliated Hospital of Sun Yat-Sen University, and informed consent was signed by the patients. The patients were offered MVV as a more permanent solution in March and July 2012, respectively.

Scrotal exploration was performed with the patients under combined spinal-epidural anaesthesia. The left-side incision $(3 \mathrm{~cm})$ of the scrotum through the tunica vaginalis was made, and the left vas deferens was delivered through this incision. The painful lumps and nerveimpinging tissue at the vasectomy site were thoroughly resected by electrocautery. Distal patency was confirmed by infusing diluted methylene blue through the abdominal side of the vas deferens, resulting in blue colouring of the urine. A $12 \times$ to $15 \times$ operating microscope (Leica Microsystems (Schweiz) AG, Heerbrugg, Switzerland) was used

${ }^{1}$ Department of Urology, Huangpu Hospital of the First Affiliated Hospital, Sun Yat-Sen University, Guangzhou 510700, China and ${ }^{2}$ Department of Urology, the First Affiliated Hospital of Sun Yat-Sen University, Guangzhou 510080, China

* These authors contributed equally to this work.

Correspondence: Dr CH Deng (dch0313@163.com)

Received: 4 March 2013; Revised: 31 March 2013; Accepted: 30 April 2013; Published online: 8 July 2013 
to visualize the field. Aspirate from the testicular end of the vasal lumen was opalescent, and spermatozoa were observed in both cases. The patients were then treated by MVV. Three mucosal sutures (double-armed, 10-0 nylon (length $300 \mathrm{~mm}$ and curvature 3/8)) were placed from inside to outside on the mucosa. After all of the mucosal sutures were tied with surgeon's knots, 8-0 prolene sutures (Ethicon W2777 (length $6.8 \mathrm{~mm}, 450 \mathrm{~mm}$ and curvature 3/8)) were placed through the muscularis and adventitia between each pair of mucosal sutures. After the anterior layer was sutured, the approximator was then rotated and folded to expose the mucosa to perform the posterior mucosal and muscularis anastomosis. Anastomosis of the muscularis and adventitial layers was completed using 8-0 prolene sutures. The incision was closed in layers. The right-side operative step was the same as the left.

The operative times were 180 and $300 \mathrm{~min}$, respectively. We did not observe any intra- or postoperative complications. The severity of pain scores, depression scores and anxiety scores was assessed during office visits and telephone interviews at 1, 3, 6 and 12 months after surgery. Seven days after the procedure, the patients reported complete remission of the pain; both pain scores were 0 point. At 6 months after the operation, the pain scores were 0 and 1 points, the depression scores were 30 and 32 points, and the anxiety scores were 30 and 29 points, respectively. The dilatation and tenderness of the caput epididymides had clearly been relieved. At the 12-month follow-up, the pain score for one patient remained at 0 point, and sperm were present in the semen $\left(\right.$ sperm count $=50.1$ million $\mathrm{ml}^{-1}$ ). At 9 months, the other patient showed a pain score of 1 point, and sperm were present (62.51 million $\mathrm{ml}^{-1}$ ). No complications, such as haematoma or testicular atrophy, occurred.

Chronic scrotal pain after vasectomy poses a unique challenge for urologists and patients. It is difficult to treat, as the cause is often unknown. The incidence of chronic scrotal pain after vasectomy has been estimated at $1 \%-19 \% .{ }^{4,5}$ Severe scrotal pain, defined as a visual analogue pain score greater than 5 , was reported in $1 \%-6 \%$ of men after vasectomy in a study that followed participants for 10 years. ${ }^{6}$ Surgical intervention should be considered only after the failure of conservative management.

MVV was considered a rational and effective treatment option for intractable chronic scrotal pain after vasectomy. Nangia et al. ${ }^{7}$ reported that $69 \%$ of patients were pain free after reversal, noting that the selection criteria for surgery are important to the outcome. In a small series, Myers et al. ${ }^{8}$ reported that $84 \%$ of patients with intractable chronic scrotal pain after vasectomy had complete resolution of pain after vasovasostomy. In our preliminary experience, the operative result also demonstrated complete pain relief during a 6- to 12-month follow-up period. We believe that the pain relief was primarily due to the MVV. Although these two patients were relative older, the procedure may be helpful for younger patients who may require future fertility and not pain relief because MVV can result in good patency. However, painful lumps were also present at the vasectomy site upon physical examination, and removal of the painful lumps and nerveimpinging tissue at the vasectomy site may have also played an important role in relieving the pain and preventing recurrence.

Microsurgical denervation of the spermatic cord can be offered to intractable patients who have complete or partial temporary relief of chronic scrotal pain following spermatic cord block using a local anaesthetic. The technique is perhaps the most interesting advance to date, with complete and durable resolution of pain reported in $71 \%-96 \%$ of cases. ${ }^{9}$ Both of our patients, who had chronic scrotal pain after vasectomy that was refractory to conservative management, were given a spermatic cord block. The patients experienced complete and temporary relief of their pain. Microsurgical denervation of the spermatic cord was also recommended for these patients, but they selected MVV as a final treatment option. Epididymectomy has a limited role in the management of intractable scrotal pain, with varied success rates. ${ }^{10,11}$ Orchidectomy should be considered as a last resort if these procedures fail.

In conclusion, intractable chronic scrotal pain after vasectomy is a rare but serious complication of vasectomy. It remains a challenging and frustrating problem for both patients and urologists. In appropriately selected patients, MVV can be a safe and effective management option for the treatment of intractable chronic scrotal pain after vasectomy.

\section{AUTHOR CONTRIBUTIONS}

XAT designed the study and performed the microsurgical procedure. LZ and LYZ performed the microsurgical procedure and drafted the manuscript. CMZ, JTZ, JQZ and KLL participated in the acquisition and analysis of the data. XZS and SPQ provided important intellectual advice and helped to revise the manuscript. CHD conceived the study and performed the microsurgical procedure.

\section{COMPETING FINANCIAL INTERESTS}

All authors declare that there are no competing financial interests.

\section{ACKNOWLEDGMENTS}

This study was supported by grants from the National Natural Science Foundation of China (Nos. 81172432, 81070488 and 81270696), Guangdong Natural Science Foundation (No. 9151008901000043), Guangdong Provincial Ministry of Cooperation Project (No. 2011B090400034) and the Scientific and Technical Project of Guangdong Province (No. 2012B031800361).

1 Tandon S, Sabanegh E Jr. Chronic pain after vasectomy: a diagnostic and treatment dilemma. BJU Int 2008; 102: 166-9.

2 Leslie TA, Illing RO, Cranston DW, Guillebaud J. The incidence of chronic scrotal pain after vasectomy: a prospective audit. BJU Int 2007; 100: 1330-3.

3 Herrel L, Hsiao W. Microsurgical vasovasostomy. Asian J Androl 2013; 15: 44-8.

4 Granitsiotis P, Kirk D. Chronic testicular pain: an overview. Eur Urol 2004; 45: 430-6.

5 Manikandan R, Srirangam SJ, Pearson E, Collins GN. Early and late morbidity after vasectomy: a comparison of chronic scrotal pain at 1 and 10 years. BJU Int 2004; 93 : 571-4.

6 Romero Pérez P, Merenciano Cortina FJ, Rafie Mazketli W, Amat Cecilia M, Martínez Hernández MC. Vasectomy: study of 300 interventions. Review of the national literature and of its complications. Actas Urol Esp 2004; 28: 175-214.

7 Nangia AK, Myles JL, Thomas AJ Jr. Vasectomy reversal for the post-vasectomy pain syndrome: a clinical and histological evaluation. J Urol 2000; 164: 1939-42.

8 Myers SA, Mershon CE, Fuchs EF. Vasectomy reversal for treatment of the postvasectomy pain syndrome. J Urol 1997; 157: 518-20.

9 Levine L. Chronic orchialgia: evaluation and discussion of treatment options. Ther Adv Urol 2010; 2: 209-14.

10 Sweeney CA, Oades GM, Fraser M, Palmer M. Does scrotal surgery have a role in management of chronic scrotal pain? Urology 2008; 71: 1092-102.

11 West AF, Leung HY, Powell PH. Epididymectomy is an effective treatment for scrotal pain after vasectomy. BJU Int 2000; 85: 1097-9. 